\title{
Ultrafast consensus via predictive mechanisms
}

\author{
Hai-Tao Zhang ${ }^{1,2}$, Michael ZhiQiang Chen ${ }^{2,3(a)}$, Tao Zhou ${ }^{4,5}$ and Guy-Bart Stan ${ }^{2}$ \\ ${ }^{1}$ Department of Control Science and Engineering, Huazhong University of Science and Technology \\ Wuhan 430074, PRC \\ ${ }^{2}$ Department of Engineering, University of Cambridge - Cambridge CB2 1PZ, UK, EU \\ ${ }^{3}$ Department of Engineering, University of Leicester - Leicester LE1 7RH, UK, EU \\ ${ }^{4}$ Department of Modern Physics, University of Science and Technology of China - Hefei 230026, PRC \\ ${ }^{5}$ Department of Physics, University of Fribourg, Chemin du Muse - CH-1700 Fribourg, Switzerland
}

PACS 05.65.+b - Self-organized systems

PACS 87.17.Jj - Cell locomotion, chemotaxis

PACS 89.75.-k - Complex systems

\begin{abstract}
An important natural phenomenon surfaces that ultrafast consensus can be achieved by introducing predictive mechanisms. By predicting the dynamics of a network several steps ahead and using this information in the consensus protocol, it is shown that, without changing the topology of the network, drastic improvements can be achieved in terms of the speed of convergence towards consensus and of the feasible range of sampling periods, compared with the routine consensus protocol. In natural science, this study provides an evidence for the idea that some predictive mechanisms exist in widely-spread biological swarms, flocks, and schools. From the industrial engineering point of view, inclusion of an efficient predictive mechanism allows for a significant increase in the consensus speed and a reduction of the required communication energy.
\end{abstract}

Over the last decade, scientists have been looking for common, possibly universal, features of the collective behaviors of animals [1], bacteria [2], cells [3], molecular motors [4], as well as driven granular objects [5]. The collective dynamics of networks of interconnected agents is currently a subject of intensive research that has potential applications in biology, physics and engineering. In this area, one of the most general and attractive topic is the consensus problem [6-8], where groups of agents agree upon certain quantities such as attitude, position, temperature and voltage. Furthermore, solving consensus problem using distributed computational methods has direct implications on sensor network data fusion, load balancing, swarm control, unmanned air vehicles (UAVs), attitude alignment of satellite clusters, congestion control of communication networks, multi-agent formation control, etc. [9-11].

One of the central problems in the study of collective dynamics is the design of consensus protocols allowing to reach consensus as quickly as possible using the least amount of communication energy. Recently, by using parallel and distributed computing approaches [12] or

\footnotetext{
(a) E-mail: mc274@le.ac.uk
}

numerical statistical analysis, some methods aiming at enhancing the consensus speed have been proposed, such as convex optimization [13], introduction of long-range interactions [14], stability analysis [15], the relation between communication intensity and convergence speed [16], design of the heterogeneous influences [17], and use of adaptive velocity [18]. Most of the previous studies on the consensus of interconnected agents has been based on the implicit assumption that an agent's state at the next discrete-time step is solely determined by the current (network) state. However, in natural bio-groups, individuals typically have some higher-level intelligence, namely predictive intelligence, which is the ability of predicting the future position of some group members based on their past and current positions. Microscopically, recent experiments on the bio-eyesight systems have revealed the predictive functions of the optical and acoustical apparatuses, especially the retina and cortex [19-21]. Macroscopically, experiments on bee groups $[22,23]$ have provided evidences for the existence of predictive mechanisms in bee swarm formation and foraging. Unfortunately, predictive mechanisms have been ignored in most prior studies of consensus. In this letter, inspired by the natural existence of predictive mechanisms, we a) design 
a consensus protocol incorporating some predictive mechanism; b) provide a mathematical framework with which the robustness properties of the proposed protocol can be demonstrated; c) illustrate, via numerical simulations, the significant improvements of the consensus speed compared with the routine protocol. This work highlights a potential predictive consensus mechanism in swarms, which is of practical significance in designing the communication and motion protocols of distributed intelligent mobile agents, such as mobile sensor networks and cooperative robots.

We represent a network of interacting agents by a digraph $G=(\mathcal{V}, A)$, where $\mathcal{V}=\left\{v_{1}, \ldots, v_{N}\right\}$ is the set of $N$ nodes representing the agents, and $A$ is the $N \times N$ adjacency matrix with $a_{i j} \geqslant 0$ denoting the edge weight from node $i$ to node $j$. No self-cycle is allowed, hence $a_{i i}=0$ for all $i$. Let $x_{i}(k) \in \mathbb{R}$ denote the state of node $i$, which could represent physical quantities such as attitude, position, temperature, voltage, etc. Generally, we say the nodes of a network have reached a consensus if and only if $x_{i}(k)=x_{j}(k)$ for all $i, j \in \mathcal{V}$ and for all $k>\bar{k}$. Whenever the nodes of a network are all in agreement, their common value is called the group decision value. If this value is $\bar{x}(0)=1 / N\left(\sum_{i=1}^{N} x_{i}(0)\right)$, the network is said to have reached the average-consensus.

We consider the following discrete-time model for the dynamics of agent $i$ :

$$
x_{i}(k+1)=x_{i}(k)+\epsilon u_{i}(k),
$$

where $\epsilon$ denotes the sampling period or step size and $u_{i}(k)$ denotes the control input. Although this model is a simple linear one in which all nodes change behaviors at the same time, it is a benchmark model $[8,14,24-26]$ to test the performance of different kinds of consensus protocols. For such dynamic models, average consensus is typically asymptotically reached using the routine consensus protocol

$$
u_{i}(k)=-\sum_{j=1}^{N} a_{i j} \Delta x_{i, j}(k), \quad(i, j=1, \ldots, N),
$$

where $\Delta x_{i, j}(k)=x_{i}(k)-x_{j}(k)$ denotes the state difference between the $i$-th and $j$-th agents [8]. The network dynamics, under the routine protocol, is

$$
x(k+1)=P_{\epsilon} x(k),
$$

where $P_{\epsilon}=I_{N}-\epsilon L$, and $L$ is the Laplacian matrix of $G$ defined as $l_{i i}=\sum_{l \neq i}^{N} a_{i l}$ and $l_{i j}=-a_{i j}, \forall i \neq j$. Denote by $d_{\max }=\max _{i}\left(l_{i i}\right)$ the maximum out-degree of $G$. If $G$ is strongly connected and balanced (i.e. $\forall i, \sum_{j} a_{i j}=$ $\left.\sum_{j} a_{j i}\right)$, and the sampling period $\epsilon \in\left(0,1 / d_{\max }\right)$, then the routine consensus protocol (2) ensures global asymptotic convergence to consensus $[8,24]$.

We now introduce a predictive average-consensus protocol for complete digraphs, i.e. digraphs for which $a_{i j}>0$,

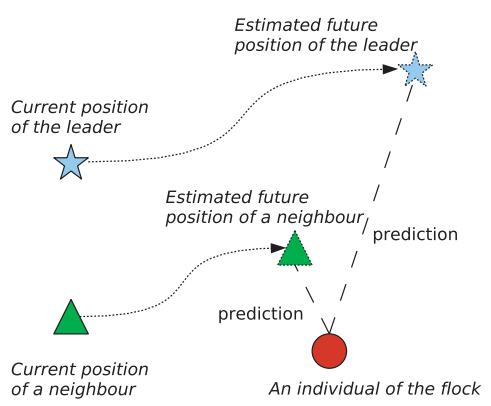

Fig. 1: (Color online) Illustration of the prediction mechanism ubiquitous in bio-groups.

$\forall i \neq j$. A general physical picture illustrating this paradigm is given in fig. 1 and interpreted as follows: in widely spread natural bio-groups composed of animals, bacteria, cells, etc., the next-step behavioral decision is not solely based on the current available state information (e.g. position, velocity, etc.) of the other, neighboring or leading, agents inside the group, but also on their predicted future states. More precisely, keeping in mind a few past states of its leader and neighbors, an agent can estimate its future states several steps ahead and then make a decision on its own next action. To integrate such mechanism into the routine protocol, we consider an additional prediction term $v_{i}(k)$ and add it to the routine control input given in eq. (2):

$$
u_{i}(k)=\sum_{j=1}^{N} a_{i j} \Delta x_{i, j}(k)+v_{i}(k) .
$$

The network dynamics are now given by

$$
x(k+1)=P_{\epsilon} x(k)+v(k) .
$$

Using the above protocol, the future states can be predicted based on the current ones $x(k)$ as indicated below:

$$
\begin{gathered}
x(k+2)=P_{\epsilon}^{2} x(k)+P_{\epsilon} v(k)+v(k+1), \\
\vdots \\
x\left(k+H_{p}\right)=P_{\epsilon}^{H_{p}} x(k)+\sum_{j=0}^{H_{p}-1}\left(P_{\epsilon}^{H_{p}-j-1} v(k+j)\right),
\end{gathered}
$$

where the integer $H_{p}$ defines the number of predicted steps. In this way, the future discrete-time evolution of the network can be predicted $H_{p}$ steps ahead as

$$
X(k+1)=P_{X} x(k)+P_{U} U(k)
$$

with

$$
\begin{aligned}
X^{T}(k+1) & =\left[x^{T}(k+1), \ldots, x^{T}\left(k+H_{p}\right)\right], \\
U^{T}(k) & =\left[v^{T}(k), \ldots, v^{T}\left(k+H_{p}-1\right)\right],
\end{aligned}
$$




$$
\begin{aligned}
P_{X} & =\left[\begin{array}{c}
P_{\epsilon} \\
\vdots \\
P_{\epsilon}^{H_{p}}
\end{array}\right], \\
P_{U} & =\left[\begin{array}{llll}
I_{N} & & \\
P_{\epsilon} & I_{N} & \\
\vdots & \vdots & \ddots & \\
P_{\epsilon}^{H_{p}-1} & P_{\epsilon}^{H_{p}-2} & \ldots & I_{N}
\end{array}\right] .
\end{aligned}
$$

Since the general goal of a consensus protocol is to eliminate the disagreements between individuals, we first calculate the state difference of the $i$-th and $j$-th agents, $m\left(1 \leqslant m \leqslant H_{p}\right)$ steps ahead, as

$$
\Delta x_{i, j}(k+m)=x_{i}(k+m)-x_{j}(k+m)=e_{i, j} x(k+m),
$$

where $\quad e_{i, j}=e_{i}-e_{j} \quad$ and $\quad e_{j}=\left[0, \ldots, 0,1^{j t h}, 0, \ldots, 0\right]$ denotes the $j$-th row-extracting operator. Based on eq. (7), the state difference vector, $m$ steps ahead, is

$$
\begin{aligned}
& \Delta x(k+m)=\left[\Delta x_{1,2}^{T}(k+m), \ldots, \Delta x_{1, N}^{T}(k+m),\right. \\
& \left.\Delta x_{2,3}^{T}(k+m), \ldots, \Delta x_{2, N}^{T}(k+m), \ldots, \Delta x_{N-1, N}^{T}(k+m)\right]^{T} .
\end{aligned}
$$

Accordingly, the future evolution of the network's state difference can be predicted as follows:

$$
\begin{aligned}
\Delta X(k+1) & =\left[\Delta x(k+1)^{T}, \ldots, \Delta x\left(k+H_{p}\right)^{T}\right]^{T} \\
& =P_{X E} x(k)+P_{U E} U(k)
\end{aligned}
$$

with $P_{X E}=E P_{X}, P_{U E}=E P_{U}, E=\operatorname{diag}(e, \ldots, e)$ and $e=\left[e_{1,2}^{T}, \ldots, e_{1, N}^{T}, e_{2,3}^{T}, \ldots, e_{2, N}^{T}, \ldots, e_{N-1, N}^{T}\right]^{T}$.

To solve the average-consensus problem, we first set the moving horizon optimization index that defines our model predictive control (MPC) consensus problem as below:

$$
J(k)=\|\Delta X(k+1)\|_{Q}^{2}+\|U(k)\|_{R}^{2},
$$

where $Q$ and $R$ are compatible weighting matrices which are generally set as $Q=q I_{H_{p} N(N-1) / 2}(q>0), R=I_{H_{p} N}$, and $\|M\|_{Q}^{2}=M^{T} Q M$. In eq. (9), the first term penalizes the state difference (the disagreement) in the network over the future $H_{p}$ steps, while the second term penalizes the additional MPC control energy $v(k)$. The minimum $J(k)$ corresponds to the condition $\partial J(k) / \partial U(k)=0$. Consequently, one obtains the optimal MPC action $v(k)=P_{M P C} x(k)$, where

$$
P_{M P C}=-\left[I_{N}, \mathbf{0}_{N}, \ldots, \mathbf{0}_{N}\right] \cdot\left(P_{U E}^{T} Q P_{U E}+R\right)^{-1} P_{U E}^{T} Q P_{X E} .
$$

Of course, large $N$ will inevitably increase the computational burden. However, no matter how large the size $N$ is, one can still compute the MPC term $v(k)$ according to eqs. (5) and (10), which will be directly distributed to each node. Thus, theoretically speaking, the MPC protocol (4) is always feasible even for networks of very large size.

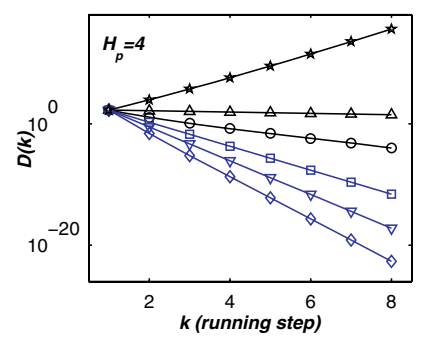

Fig. 2: (Color online) Time evolution of the instantaneous disagreement $D(k)$ of the routine (black) and MPC (blue) protocols on a symmetric, complete network of $N=10$ nodes. Here, $l_{i j}=-1(i \neq j)$ and $l_{i i}=N-1$. Case $1: \epsilon=1 / 9=1 / d_{\max }$, $\diamond:$ MPC, $\bigcirc$ : routine protocol; Case $2: \epsilon=0.03<1 / d_{\max }, \nabla$ : MPC, $\triangle$ : routine protocol; Case $3: \epsilon=2.00>1 / d_{\max }, \square:$ MPC, $\star$ : routine protocol. In the MPC protocol, $H_{p}=4, q=2$, and the initial state $x_{i}(0)$ are selected randomly in $[0,15]$.

The proposed algorithm is based on (9) and shows some consistency with the routine protocol (2). More precisely, the latter is solely based on the current state difference $\Delta x_{i, j}(k)$ of each pair in the network, while the former roots not only in $\Delta x_{i, j}(k)$ but also in the future state . difference $\Delta x_{i, j}(k+m)$. This new consensus algorithm is characterized by four important properties: i) for the case of symmetric (i.e. $a_{i j}=a_{j i}$ ), complete networks, the state matrix $P_{\epsilon}+P_{M P C}$ is also symmetric and has the same eigenvectors as the matrix $P_{\epsilon}$; ii) for the case of complete, balanced networks, the matrix $P_{M P C}$ is also balanced in the sense that $P_{M P C} \mathbf{1}=P_{M P C}^{T} \mathbf{1}=\boldsymbol{0}$ with $\mathbf{1}=[1, \ldots, 1]_{N \times 1}^{T}$ and $\boldsymbol{O}=0 \cdot \mathbf{1}$; iii) for the case of complete, balanced networks, average consensus will be achieved if and only if the matrix $P_{\epsilon}+P_{M P C}$ has a simple eigenvalue at 1 and all its other eigenvalues inside the open unit circle; iv) for the case of complete, balanced networks, if you denote each entry of $P_{\epsilon}+P_{M P C}$ by $\beta_{i j}(i, j=1, \ldots, N)$, then average consensus will be achieved, provided that $\beta_{i j} \geqslant 0(i \neq j)$ and $\beta_{i i} \in(0,1]$. The mathematical proofs of these properties are beyond the scope of this letter and will thus be published elsewhere. As a consequence to the above properties, the proposed MPC protocol yields the following two improvements compared with the routine protocol: $(\boldsymbol{A} \mathbf{1})$ the feasible range of the sampling period $\epsilon$ (i.e. the set of values of $\epsilon$ leading to average consensus) is remarkably expanded; $(\boldsymbol{A} \mathscr{2})$ for feasible values of $\epsilon$, the convergence speed is significantly improved.

To illustrate the advantages of the MPC protocol, we present some numerical results comparing the convergence speeds of the MPC and routine protocols. Since the objective is to reach average consensus, the instantaneous disagreement index is typically set as $D(k)=\|x(k)-\mathbf{1} \bar{x}(0)\|_{2}^{2}$ (here $\left.\|x\|_{2}=\left(x^{T} x\right)^{1 / 2}\right)$ and the consensus steps $T_{c}\left(D_{c}\right)$ can be defined as the number of steps required for $D(k)$ to reach a small threshold value, $D_{c}$. A reasonable measurement of the consensus speed is given by $1 / T_{c}\left(D_{c}\right)$. As shown in fig. 2 , the addition of the predictive mechanism defined in (10) yields a drastic 


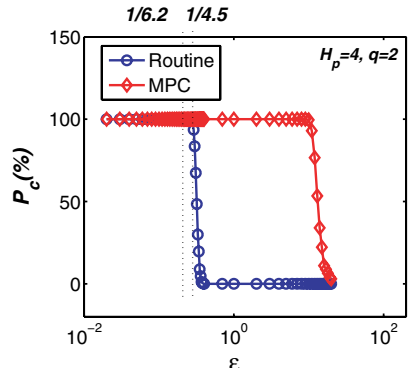

(a)

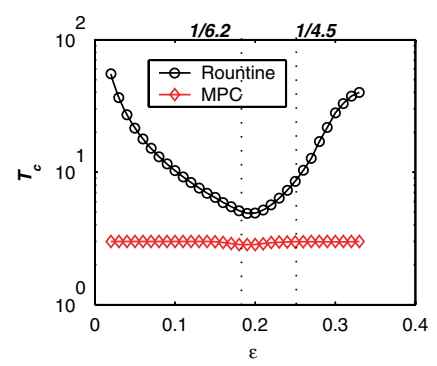

(b)
Fig. 3: (Color online) (a) Ultrafast convergence probability $\left(P_{c}(0.01)\right)$; (b) Average consensus steps $\left(T_{c}(0.01)\right)$. Comparison is addressed between the MPC and routine protocols with different $\epsilon$ and 500 independent runs for each value of $\epsilon$. For these simulations, $H_{p}=4, q=2, D_{c}=0.01$, entries $l_{i j}(i \neq j)$ of $L$ are selected randomly in $[-1,0)$ such that the resulting network is complete and balanced, and $x_{i}(0)(i=1, \ldots, N)$ is selected randomly in $[0,15]$. The associated values of $d_{\max }$ lie in $[4.5,6.2]$. The vertical dotted lines correspond to the minimum and maximum values of $1 / d_{\max }$.

increase in convergence speed. In particular, for $\epsilon<1 / d_{\max }$ $\left(d_{\max }=\max _{i}\left(l_{i i}\right)\right)$, the convergence speed is increased more than 20 times (measured by the slope of $D(k)$ curve) by using the proposed MPC protocol. Furthermore, even when the routine convergence condition is violated, i.e. $\epsilon>1 / d_{\max }$, it is observed that the MPC protocol still allows asymptotic convergence with high speed.

To further compare the performances of the MPC and routine protocols, we examine in fig. 3(a) the ultrafast convergence probability $P_{c}\left(D_{c}\right)$ with respect to $\epsilon$ on complete, balanced networks. Here $P_{c}\left(D_{c}\right)$ denotes the ratio of ultrafast convergence runs over a total of 500 runs. A convergence run is defined as ultrafast consensus if a very small value of $D_{c}$ of the disagreement index $D(k)$ can be reached within a short period of $Z$ steps. Here, we set $D_{c}=0.01$ and $Z=100$. The specific values of $D_{c}$ and $Z$ are unimportant since qualitatively the results are invariant to those values. In fig. 3(a), it can be observed that, with the assistance of predictive mechanisms, $P_{c}(0.01)$ is significantly increased, especially when $\epsilon>1 / d_{\max }$. As a consequence, the maximum feasible ultrafast convergence sampling period $\left(\epsilon_{\max }\right)$ is sharply increased (more than 40 times), which nicely illustrates the improvement $\boldsymbol{A} \boldsymbol{1}$ of the MPC protocol. Moreover, to study the effects of the predictive mechanism on the feasible convergence range of $\epsilon$, we examine in fig. 3(b) the average consensus steps $T_{c}(0.01)$ of these two strategies with different $\epsilon$. In this comparison, for each value of $\epsilon, T_{c}$ denotes the average consensus steps over all the successful convergence runs. It can be seen that, compared with the routine protocol, the MPC protocol allows for a significant increase in the consensus speed $T_{c}^{-1}$ (by a factor between 6 and 20 in our simulations), which illustrates the improvement $\boldsymbol{A} \mathscr{2}$.

It is well known that the eigenvalue distribution of the state matrix $P_{\epsilon}+P_{M P C}$ is closely related to the
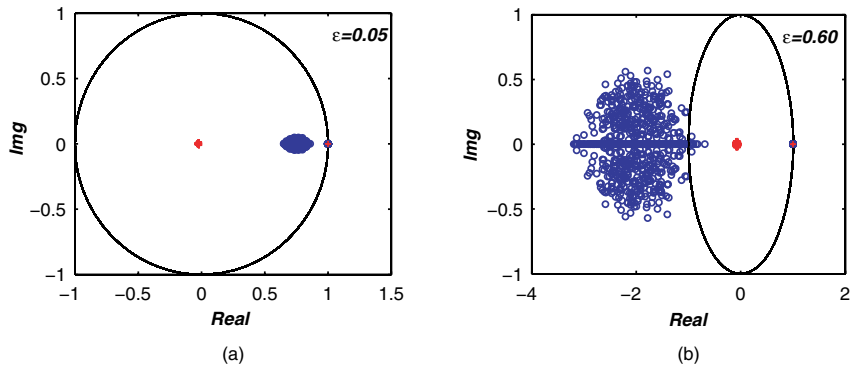

Fig. 4: (Color online) Eigenvalue distributions for different $\epsilon$. Blue $(\bigcirc)$ and red $(+)$ represent the cases of $P_{\epsilon}$ and $P_{\epsilon}+P_{M P C}$ over 100 runs, respectively. The black circle denotes the unit circle in the complex plane. Here, $H_{p}=4, q=2$, and each entry $l_{i j}(j \neq i)$ of $L$ is chosen randomly in $[-1,0)$ such that the resulting network is complete and balanced. The associated values of $d_{\max }$ lie in $[4.5,6.2]$.

convergence speed of the associated consensus protocol. Therefore, we display the eigenvalue distributions of $P_{\epsilon}$ and $P_{\epsilon}+P_{M P C}$ in fig. 4 . With these eigenvalue distributions, we can interpret the advantages of the MPC protocol more lively. Indeed, since the eigenvalue cluster of $P_{\epsilon}+$ $P_{M P C}$ is always much smaller and closer to the origin of the complex plane than the cluster of $P_{\epsilon}$, the MPC protocol generally exhibits faster consensus. When $\epsilon<1 / d_{\max }$ $(\epsilon=0.05$, see fig. 4(a)), the two eigenvalue clusters are both kept inside the asymptotic stability region, i.e. the unit circle in the complex plane. However, since each eigenvalue of $P_{\epsilon}+P_{M P C}$ is much closer to the origin, the consensus speed is sharply increased in the case of the MPC protocol. Furthermore, when $\epsilon>1 / d_{\max }(\epsilon=0.60$, see fig. $4(\mathrm{~b}))$, some of the eigenvalues of $P_{\epsilon}$ start escaping the unit circle, making the disagreement function diverge, whereas all the eigenvalues of $P_{\epsilon}+P_{M P C}$ remain inside the unit circle, which guarantees its asymptotic convergence.

In summary, motivated by the ubiquity of predicting abilities in natural bio-groups, we incorporated some predictive mechanism into the routine consensus protocol and yielded a novel MPC protocol. We presented analytical results as well as numerical simulations to demonstrate its great advantages. The comparisons between the MPC and routine protocols on complete, balanced networks led to the following two conclusions: i) just a little vision into the future can produce significant increases in convergence speeds; ii) the feasible sampling period range can be broadly expanded using this predictive mechanism, giving the MPC consensus protocol the potential to effectively save communication energy. These advantages have been explained through an eigenvalue distribution analysis. More precisely, due to the use of a prediction mechanism, the eigenvalue cluster of the state matrix is compressed and driven back towards the origin of the complex plane even when the sampling period exceeds the routine convergence threshold. 
Furthermore, to verify the generality of these conclusions, we have applied the MPC protocol to partially linked networks. Analogously, each individual predicts its neighbors' future behaviors according to their historical trajectories (past state values), and makes its own decision based on a minimization of the neighboring state difference. The corresponding results for partially linked networks are qualitatively similar $(\boldsymbol{A} \boldsymbol{1}$ and $\boldsymbol{A} 2$ are also valid in this case), except that the performance improvements are slightly reduced due to the information flow constraints due to the network topology, which is quite reasonable. On the other hand, according to eqs. (5) and (10), one may argue that the global information of the whole network including the state matrix $P_{\epsilon}$ and the current state $x(k)$ is required for each individual's prediction. This, however, is not true, and it can be proven that sufficiently long local information sequence observable for each individual is capable of constituting the local prediction mechanism for each individual. Consequently, the present predictive protocol can be implemented in a decentralized way, which further improves its generality. More detailed reasoning is provided as follows. Let $Z_{i}(k)=\left[z_{1, i}^{T}(k), \ldots, z_{i N, i}^{T}(k)\right]^{T}$ denote the $N$-length historical state sequence for the $i$-th individual with $z_{l, i}(k)=x_{i}(k+1-l),(l=1, \ldots, N)$, then the state of an arbitrary neighbor $j$ of $i$ can be predicted one step ahead by $x_{j}(k+1)=B_{j, i} Z_{i}(k)$ with $B_{j, i}=e_{j} P_{\epsilon}^{N+1} \Phi_{i}^{-1} \quad$ and $\quad \Phi_{i}=\left[\left(e_{i} P_{\epsilon}^{N}\right)^{T}, \ldots,\left(e_{i} P_{\epsilon}\right)^{T}\right]^{T}$. Fortunately, it can be proven that sufficiently long historical information sequence observed by individual $i$ can yield accurate estimate of $B_{i j}$. Therefore, local information is qualified to yield accurate prediction. For brevity, we omit the mathematical analysis of the decentralized implementation.

Still worth mentioning is that our MPC protocol is not just confined to linear networks. For networks governed by nonlinear dynamics, MPC also has the potential to yield nice performances since it can make full use of the widely-spread individual predictive intelligence to produce coordinated collective behavior [27]. Actually, in another paper [28], we have also examined the capability of predictive mechanism for two popular nonlinear flock models, the Vicsek model [5] and the A/R model [29]. The corresponding results show that the MPC protocol outperforms the routine protocol in terms of the consensus speed and the communication cost. For natural science, the contribution of this work lies in its ability to explain why members of biological swarms such as firefly and deep-sea fish groups do not communicate very frequently all along but just now and then during the whole dynamic process. From the industrial application point of view, the value of this work is that the consensus speed can be significantly enhanced with remarkably reduced communication energy or cost. All these merits are only at the expense of giving the agents capability of making predictions. In real-world applications, when it is difficult to add controllers to all nodes for multi-agent systems with large $N$, one possible approach is to use the pinning control technique [30]. This work is a first attempt aimed at achieving ultrafast consensus by adding a prediction mechanism to the classical protocol, which may open new avenues in that fascinating field.

Thanks are due to Prof. GuAnrong Chen (City University of Hong Kong) and Prof. JAN MACIEJOWsKI (Cambridge University), who offered many valuable suggestions, and the reviewers for improving the quality of this paper. H-TZ acknowledges the support of NNSFC under grant No. 60704041. TZ acknowledges the support of NNSFC under grant No. 10635040. G-BS acknowledges the support of the EPSRC under grant No. EP/E02761X/1.

\section{REFERENCES}

[1] Martínez S., Cortés J. and Bullo F., IEEE Control Syst. Mag., 27 (2007) 75.

[2] Budrene E. O. and Berg H., Nature (London), 376 (1995) 49.

[3] You L., Cox III R. S. and Weiss R., Nature (London), 428 (2004) 868.

[4] Astumian R. D. and Bier M., Phys. Rev. Lett., 72 (1994) 1766.

[5] Vicsek T., Czirók A., Ben-Jacob E., Cohen I. and Shochet O., Phys. Rev. Lett., 75 (1995) 1226.

[6] Grégoire G. and Chaté H., Phys. Rev. Lett., 92 (2004) 025702 .

[7] Aldana M., Dossetti V., Huepe C., Kenkre V. M. and Larralde H., Phys. Rev. Lett., 98 (2007) 095702.

[8] Olfati-Saber R. and Murray R., IEEE Trans. Autom. Control, 49 (2004) 1520.

[9] Akyildiz I. F., Su W., Sankarasubramaniam Y. and Cayirci E., Comput. Netw., 38 (2002) 393.

[10] Ogren P., Fiorelli E. and Leonard N. E., IEEE Trans. Autom. Control, 49 (2004) 1292.

[11] Arai T., Pagello E. and Parker L. E., IEEE Trans. Robot. Autom., 18 (2002) 655.

[12] Bertsekns D. P. and Tsitsiklis J. N., Parallel and Distributed Computation: Numerical Methods (Prentice Hall) 1989.

[13] Xiao L. and Boyd S., Syst. Control Lett., 53 (2004) 65.

[14] Olfati-Saber R., Proc. Am. Control Conf., 4 (2005) 2371.

[15] Moreau L., Proc. IEEE Conf. Decision Control, 4 (2004) 3998.

[16] Carli R., Fagnani F., Speranzon A. and Zampieri S., Proc. Am. Control Conf. (2006) 4189.

[17] Yang W., Cao L., Wang X. F. and Li X., Phys. Rev. E, 74 (2006) 037101.

[18] Li W. and Wang X. F., Phys. Rev. E, 75 (2007) 021917.

[19] Gottraied J. A., Doherty J. O. and Dolan R. J., Science, 301 (2003) 1104.

[20] Summerfield C., Egner T., Greene M., Koechlin E., Mangles J. and Hirsch J., Science, 314 (2006) 1311. 
[21] Melcher D., Nat. Neurosci., 10 (2007) 903.

[22] Woods E. F., Nature (London), 184 (1959) 842.

[23] Montague P. R., Dayan P., Person C. and Sejnowski T. J., Nature (London), 377 (1995) 725 .

[24] Olfati-Saber R. and Murray R., Proc. IEEE, 95 (2007) 215.

[25] Ren W., Beard R. W. and Arkins E. M., IEEE Control Syst. Mag., 27 (2007) 71.
[26] Wu Z. P., Guan Z. H. and Wu X. Y., Physica A, 379 (2007) 681.

[27] Camacho E. F. and Bordons C., Model Predictive Control (Springer) 2004.

[28] Zhang H. T., Chen M. Z. Q. and Zhou T., arXiv 0709.0380 (2007).

[29] Gazi V. and Passino K. M., IEEE Trans. Autom. Control, 48 (2003) 692.

[30] Zhou J., Lu J. and Lu J., Automatica, 44 (2008) 996. 